\title{
EIN LIED VON SANT GROBIAN.
}

\author{
Ein fcho̊n Reygenlied \\ von Sant Grobian | in der wyß | \\ Můter | můter wie fol \\ ich thůn.
}

[aj b]

5 .Dann es ift von Sant Grobian, den yetz wil fyren yederman!, Sant Grobian!

2 Sant Grobhart ift ein wůlter man, er hat ein groffen orden |:

10 Es zücht jm zů fuhier yederman, der brüderen find vil worden.

Es dienend dmann jm nit allein:

ouch frowen | alt | jung | groß vnd klein |, Sant Grobian!

153 Man eert jnn yetzund wyt vnd breyt, (fin lob ift wyt erfchollen|)

Mit fuillen | prassen | trunckenheyt: das thund die truncknen trollen. Ie einer trinckt dem andren $z \mathfrak{u}$,

20 biß sföller werdend denn ein ků ।, Die Grobian!

4 Sin Kilch das ift das wirtes hus, da thuit man jnn vereeren । Mit trincken vnd mit libben im fuß,

25 mit fpilen vnd mit fchweeren, | Mit fchamper wyß | gebård | vnd wort, die man yetz trybt an allem ort

Dem Grobian. 
aij[a] 5 Wenn fy fön in die kilchen gan

30 ir \&mpter ze volbringen $\mid$,

So zücht der Meßner dfüw glock an;

die hôrend fy denn klingen |:

Denn rennendts zů mit gantzer macht,

es fyg by tag | es fyg by nacht |

dem Grobian!

6 Grobhart die moren mit jm fưrt

mit hoffenlichen fitten |:

Wenn er ly by den oren rürt

thuit fy die fchâll erfchütten.

40 Den vortantz hat allein die mor:

der nimpts bym fchwantz|difer bym or |,

Herr Grobian!

7 Da fichft du manchen groben wan

an disem wiffen reygen |,

45 Der dfüwglock vaft wol lüten kan, von pfaffen vid von leygen |.

Doch welcher kan der grobeft fin den fchrybt der Bruder meyfter yn |

Dem Grobian.

508 Wo fich thåt famlen dife rott, da mag der tiifel lachen|!

Dann fchmånend fy vnd leftrend Gott, fy trybend wüfte lachen |:

All zucht vnd eer hat da ein end,

all fromkeit fy für torheyt hend।, Die Grobisn!

[a ij b] 9 Die mor die fol den krantz vff han, dann ir ift wol gelungen |:

Sy hat jrm hußwirt Grobian

60 yetz nliwlich bracht vil jungen.

der groben fchwyn find yetzund vil

die haltend weder maß noch zil, die Grobian!

10 Suber ins dorff ift worden blind

65 Spürt man in allen dingen:

das fchafft das dpuren truncken find।, Deu morentantz fy fpringend!

der ülpekůntz den vortantz hat ।

ift wưft genig vnd felten fatt, |

37 hoffenlichem $B . \quad 48$ růder Bmeyfter $B$. 
11 Die Ef.ls büchfen bringt er hår

die wil er felber tragen |,

Die ift gefüllt mit affen fchmår :

darnach thut mancher fragen;

75 ye einer ftrychts dem andren an, den er im orden gern wolt han |

Der Grobian.

12 Wenn man die brůderfchafft wil bgan nach irer ordenunge|,

80 So hept die Su die Mette an, die wirt fo lat gefungen:

Die Primzyt ift ins Efels thon, die Tertz můß glych harnaher gon |

Dem Grobian.

aiij[a] 8513 Hůtmacher knecht fingend die Sext. nun merckend fürbaß mere:

Von groben filtzen ift der text|! mit vollen büchen fchwåre Hept an die volle rott die Non: ichlemmer vnd demmer darzu gond।

Dem Grobian!

14 Darnach die Su zur vefper klinckt mit jrer groffen glocken |; Die brüder dife fach recht dunckt, 95 wenn jnn die Su thit locken |. Complet fingend fy allo voll der lilpekůntz vnd truncken troll, Die Grobian!

15 All voll |, all voll ift jr gefang

100 mit kotzen vnd mit koppen ||

An hals ein giten frarcken ftrang, das mul mit kadt verftopffen:

Das ghört den groben füwen zů, fo hett man etwan vor jnn růw, Vorm Grobian!

16 Es durt mich nun der gute wyn der durch fy wirt verloren; Wol möchtend dmütren trurig fin die follich fün hand gboren |:

110 Wo folich lit nit trunckend wyn, der kopff gult kum ein ortelin;

Der Grobian! 
[aiij b] 17 Grobhart der helg ift kumen $\nabla B$, ift worden gantz gemeine,

115 Man findt jnn yetz in manchem huß. merckend wie ichs es meyne:

Vernunfft vnd witz man wenig trybt, grob ifts was man yetz redt vnd fchrybt Vom Grobian!

12018 Von wưften dingen man yetz feyt, hört man in allen fachen:

Man fchont nit Gotts noch erberkeyt! des thuind die narren lachen. Welch brůder kan der wưfteft fin, 125 dem büt man bald ein glaß mit wyn । Dem Grobian!

19 Sant Grobhart ift in aller zunfft mit Schamper wyß vnd worten |, Sy bruchend vaft wenig vernunfft

130 dunckt mich an allen orten |: Des Efels fchmaltz vnmůflig ift, mit moren fohmaltz ift es vermifeht ।

Dem Grobian!

20 Der yetz vil füwerck tryben kan

135 vud fchamper vnzucht leeren, | Den halt man für ein werden man vnd fchrybts jm zhohen eeren |: Man bitt jnn das er noch eins fag, man lacht fin daß das huß erwag | Des Grobian!

[aiiij a] 21 Man fpricht: das ift elh gater fchwanck, deß mo̊chtind wir gelachen ।;

Darby wirt vns die zJt nit lang. was woltend wir funft machen|?

145 'Es ift kein Gott, | es ift kein hell; dem pfaffen gloub welcher da well', ।

Spricht Grobian!

22 'Von keinem hand wir nye gehơrt, der von der hell fyg kommen।;

150 Vnd wie es dôrt fyg ein gefert, wår hat das ye vernommen? Gfellfchafft tryben ift nit fünd, die pfaffen lagend was fy wend, |' Spricht Grobian. 
15523 'Nun lond vas git gefellen fin, klein zyt hand wir vff erden, Füllen vnd praffen by dem wyn: was mag vns funft mee werden !? $W_{a r}^{e}$ ftirbt der ift doch fchon da hin, 160 nach mtod ift weder fröud noch pyn, |' Spricht Grobian.

24 Der Gottloß man trybt diß gefert, die groben kůntzen alle |;

Vnd was in difen orden gehơrt,

165 die trybend jren fichalle:

All welt fy fchendend | vor ab Gott der fin fan für fy ggeben hat, I

Merck Grobian!

[a iiij b] 25 Ir jungen, wend jr hôren mich

170 vnd volgen miner leere |:

Der zucht vnd fromkeyt flyffend üch, das bringt üch lob vnd eere |!

Kein ding der jugend bas ftadt an, denn züchtig wyß ond bürden han |; Merck Grobian!

26 Ir jungen, huitend üch daruor: lond ïch di $\beta$ volck nit btriegen |: Gott ift in finr verheyffung waar, er kan noch mag nit liegen;

180 Die bôfen gond in ewig Itraaff, Zum himmel gho̊rend gottes fcharff!

Merck Grobian!

Ich far daruon.

Das vorstehende lied ist in einem einzeldruck der königl. bibliothek zu Berlin (signatur: Ye 401) erbalten, der ursprunglich der Meusebachschen sammlung angehörte. Vermutlich bei ibrer katalogisierung lernte Zarncke dieses Reygenlied kennen und veröffentlichte von ihm später in seiner ausgabe des Narrenschiffes (CXX) zwei strophen. Er gab hier bereits den nachweis, dass in der hauptsache das 72. capitel der Brantschen dichtung bearbeitet sei. Zarnckes publication folgte der abdruck Franz M. Böhmes in seinem Altdeutschen liederbuch (no. 313). Da Zarncke im Narrenschiff indes nicht die herkunft des liedes angegeben hatte, musste Böhme sich mit den beiden mitgeteilten strophen 
begntigen. Auch Hauffen (Casp. Scheidt, QF. 66, 23) scheint das lied nur durch Zarnckes bemerkungen bekannt geworden zu sein. ${ }^{1)}$ Einer kürzlich an mich gerichteten anfrage Böhmes nach dem aufbewahrungsort des druckes nachgehend, gelang es mir durch die liebenswürdigkeit der Berliner königl. bibliothek ihn dort nachzuweisen. Das lied verdient es, als ausfluss einer interessanten zeitströmung und wegen seiner frischen und nicht ungewanten darstellung durch einen abdruck allgemein zugänglich gemacht zu werden.

Das Reygenlied von Sant Grobian ist, wie schon Zarncke a. a. o. erwähnt, in einem drucke ohne ort und jahr erhalten. Unter dem titel stehen zwei zahlen (wol 57; ein 26 scheint es mir nicht zu sein), anscheinend mit dinte von einer älteren hand geschrieben. Zarncke setzt den druck um die mitte des 16. jahrhunderts und wird wol damit recht haben. Der druck umfasst vier signierte (a ij, a iij) blătter. Auf der ersten seite befindet sich nur der titel, auf den tubrigen stehen je vier strophen; doch auf der letzten nur zwei.

Wenn zwar die heimat des druckes ebensowenig wie die zeit bekannt ist, so lässt sich doch mit grosser wahrscheinlichkeit vermuten, dass er dem gebiete des Oberrheins (Elsass oder Basel, das ja sprachlich nicht viel von Strassburg differiert) angehört und sich nicht weit von dem entstehungsort des gedichtes selbst entfernt.

Dass unser lied aber in der heimatslandschaft Brants und Murners entstanden ist, zeigen deutlich die sprachformen, wie sie in den reimen und im versinnern erscheinen. Ihr gleichartiges auftreten an beiden stellen veranlasst mich eben auch, den gleichen entstehungsort fur das lied und seinen druck anzunehmen. Einige hauptcharakteristica fuhre ich an und bitte Zarnckes ausgabe des Narrenschiffes s. $268 \mathrm{ff}$. zu vergleichen.

Von reimbindungen mache ich namhaft auf dem gebiete des vocalismus: vereeren : fchweeren (iurare) 23 , mere (mêre) : fchwåre 86, här (her) : mår 1, gehört : gefert 148. 162, daruor : waar 176, fitten : er/chütten 37, mich : üch 169. Eine erklärung

1) Hauffen erwähnt unser lied l. c. s. 23, gibt aber in der anmerkung ein durch verwechslung entstandenes falsches citat: es sind bei den anmerkungen die zahlen 11 und 12 zu vertauschen.

Beiträge zur geschichte der deutschen spraobe. XVIII. 
erfordern wol die bindungen klünckt: dunckt 92, was ich als mhd. klenkt : dünkt und nicht als klingt : dünkt auffasse ${ }^{1}$ ), und fünd: wend 152. Mundartlich trat bei en und ün ein wandel in den gleichen vocal (meist $\ddot{a}$ ) ein (vgl. z. b. Mankel, Strassburger studien 2,117). Ein epithetisches $e$ im reime zeigt sich bei alle : fchalle (acc. sing.) 163. Endlich hin:pyn 159.

Auf dem gebiete des consonantismus: ift : vermi/cht 131 (vgl. ift : vermi/cht Brant 72,58), koppen: verstopffen 100. Hier ist wol koppen in kopfen zu hessern, eine nebenform, die fur eine andre bedeutung des gleichen wortes das $\mathrm{DWb} .5,1790,3$ als köpfen belegt (vgl. auch das Murnersche köpfelsknaben gegenuber sonstigem köppelsknaben DWb. 5, 1789).

Wie die reime reygen : leygen 44 , so zeigt auch das innere des verses die entwickelung eines $g$ : es syg 34. 149. Den tberschuss eines $t$ bieten die reime bei dem plural praesentis des verbums dringen: fpringend 65 , Non : gond 89 . Die formen auf -ent bietet auch das versinnere.

Von anderen formen erwähne ich noch: fy fagend (conj. praes.) 153, sy hend (: end) 54, sy fónd 29 (neben follend 4), ir wend 169, sy wend (: füd) 153. Weiter: kilch 22. 29, helg (heilige) 103, harnaher 83 neben hår z. b. 71 (: /chmär), nun (nur) 106. Aus der nominalflexion nenne ich den nom. plur. dmútren (die mutter) 108 und den gen. plur. der brúderen (der brtider) 11.

Das durfte genllgen, um die elsăssische herkunft des dichters und druckers $z u$ zeigen. Einem elsässischen poeten verdankt unser verfasser auch die anregung und die unterlage zu seinem liede.

Zarncke sagt (a. a. 0.) sicher richtig, dass unser lied eine bearbeitung des 72. capitels von Brants Narrenschiff sei. Allein damit wird man seinen verdiensten kaum ganz gerecht. Es ist keine blosse neue versification, sondern das tatsăchliche material ist durch manche zutaten bereichert und geschickt in

1) Ich nehme klünckt nicht als klingt, trotzdem an der entsprechenden stelle bei Brant (v. 54) gerade dieses wort sich findet. Wenn nicht der reim fünd : wend ebenfalls aufträte (bei Brant stünd: sünd, went : geschend $\nabla .83$ ff.) könnte man eher, selbst bei der im liede auftretenden orthographie, an die abgelehnte annahme denken. 
eine neue form gegossen. Das lied zeigt in seinem rhythmus schwung und atmet in seinen meisten partien Murnerschen geist. Es hat einen frischeren gang und freieres leben als die darstellung im Narrenschiff, die zwar eine der besten partien des Brantschen werkes und an guten ideen reich ist, der aber trotzdem in der art des stils und der diction, um mit Möricke zu reden, 'ein schulschmäeklein' anklebt.

Der verfasser des liedes hat im allgemeinen bei seinem umgiessen den inhalt des Brantschen capitels verbreitert. Er sagt statt in einem, in $z w e i$ parallelsätzen dasselbe, er verwertet manchen gedanken Brants doppelt in verschiedenartigen verknupfungen, er gebraucht fur ein verweisendes pronomen einen umschreibenden satz und macht oft eine gleichgiltige, streng genommen uberflussige bemerkung des reimwortes wegen. Aber trotz allem zeigen seine verse fall und schwung. Neue, gltickliche einfälle sind aus andeutungen Brants hervorgegangen, aus zufällig auftretenden worten eigenartig gebildet worden. Der bearbeiter folgt durchaus nicht immer dem gang des Brantschen capitels, sondern hat seinen inhalt in sich aufgenommen und verarbeitet ihn an vielen stellen selbständig. Das wird hoffentlich die folgende einzelbetrachtung zeigen.

Gleich der eingang bietet eigenes: in der frischen weise des volksliedes und mit alten wolbekannten formeln manche volkstlimliche erinnerung wachrufend, rauscht er dahin. Erst v. 5 nimmt das thema auf und entspricht den beiden anfangsversen des Brantschen capitels. Die zweite strophe ist ganz selbständig im geiste des liedes gedichtet und auch in den beiden folgenden sind nur geringe andeutungen aus Brant entlehnt:

\section{Brant:}

1 Eyn nuwer heylig heiffut Grobian

Den will yetz fyren yederman

Vnd eeren jnn an allem ort

Mit schåntlich wi̊st werck, wis, vnd wort. lied:

5 Dann es ift von Sant Grobiau den yetz wil fyren yederman.

15 Man eert jnn yetzund wyt vnd breyt.

(Man verehrt ihn)

26 Mit Schamper wyß, gebård vnd wort,

die man yetz trybt an allem ort Dem Grobian.

Das ganze bild, dass die Grobianer in ihrer kirche (im wirtshaus) die horen begehen, zu denen das läuten der sau- 
glocke ruft, ist genauer durchgefthrt und weiter ausgemalt als bei Brant; so in strophe 4 und 5. In strophe 6 sind wider mehr ubereinstimmungen:

8 Der narr die suw byn oren hat Schuitt sie das jr die suwglock kling.

11 Die suw hat yetz alleyn den dantz,

Sie hålt das narrenschiff bym fchwantz.

36 Grobhart die moren mit jm färt Wenn er fy by den oren rürt thuit fy die fchåll erfchütten.

40 Den vortantz hat allein die mor: der nimpts bym fehwantz, difer bym or.

Hier sieht man die wirkung solch rein formaler zufälligkeiten: das wort /chwantz bei Brant fuhrt auf die v. $41 \mathrm{zu}$ grunde liegende vorstellung. Die strophen 7 und 8 sind in der hauptsache frei zusammengesungen; der dichter folgt andeutungen bei Brant, in v. $21 \mathrm{f}$. (Wer wol die sunglock lüten kan, der múffz yetz syn do vornan dran) und im sinne der folgenden verse enthalten, vermeidet aber gltucklich die dort paradierenden beispiele und classischen reminiscenzen.

Die suw alleyn die kron vff hett (NS. 72, 20) und Aber die fun macht yetz vil jungen (NS. 72,17) gibt unserm dichter das bild von der sau an die hand, die den kranz bekommt, weil sie ihrem hauswirt Grobian neulich viel junge geboren hat. Die idee dieses seltsamen paares ist neu. Die 10. strophe entspricht mit geringen veränderungen den versen $31-34$ bei Brant. Unter den zusätzen ist als glucklich zu erwähnen, dass die grobianer den morentanz springen, eine anspielung auf môr (schwein) und mit anlehnung an den bekannten morischkentanz; ganz ähnlich wie Brant (a. a. o. v. 10) sagt, dass die sau dem narren den Moringer singt.

Die 11. strophe zeigt wider das eklektische und ausgestaltende verfahren des verfassers; so z. b. ist $\mathbf{v} .72$ aus dem Brantschen verse 36 und v. 74 aus Brant 39 entstanden:

\section{Brant:}

lied :

35 Eyn yeder narr will suw werck triben 71 Die Efels büchfen bringt er hår

Das man jm lofz die blichsen bliben

Die man vmbfürt mit esels fchmer

Die esels büchs wïrt seltten ler

Wie wol eyn yeder dryn will griffen.

60 Das stricht eyn gsell dem andern an

Den er will jn der gsellfchaffit han die wil er felber tragen, Die lift gefullt mit affen fchmår : darnach thuit mancher fragt; ye einer ftrychts dem andren an, den er im orden gern wolt han Der Grobian! 
Strophe 12 hat so ziemlich ibre entsprechungen bei Brant v. $46--49$, str. 13 in v. $50-53$, strophe 14 in v. 54,56 und 57. Dagegen ist str. 15 freier behandelt und nur aus v. 57 hervorgegangen. Die 16. strophe greift wider zurück auf den anfang bei Brant; auch entsprechen sich nur Brant v. $15 \mathrm{f}$. = lied $110 \mathrm{f}$. Ganz glicklich macht unser dichter aus der Brantschen Grobheyt einen heiligen Grobhart, den auch Scheidt (Grobianus 2678; vgl. auch Hauffen a. a. o. s. 22) kennt. Sonst entsprechen ziemlich genau Brants verse 41-44 der strophe 17, ebenso Brant 6366 der strophe 18. In der folgenden strophe sind nur die schlussverse $131 \mathrm{f}$. $=$ Brant $58 \mathrm{f}$. Aus Brants versen $65,67 \mathrm{f}$. ist str. 20, aus 69, 70, 74 str. 21 geworden. Genau correspondiert Brants versen $81-85$ str. 22, Brant 76-80 str. 23. Aus andeutungen Brants (hauptsächlich 92-94) ist str. 24 entstanden, während die moralisierenden, sich direct an die jugend wendenden beiden letzten strophen keine entsprechung bei Brant zeigen, wenn man nicht in dem letzten verse des 72. capitels fur sie eine anregung finden will.

HALLE A. S., januar 1894.

JOHN MEIER. 